\title{
Simulating Marvel with the Stun Code
}

\author{
L. A. Glenn
}

\section{May 23, 2001}

U.S. Department of Energy

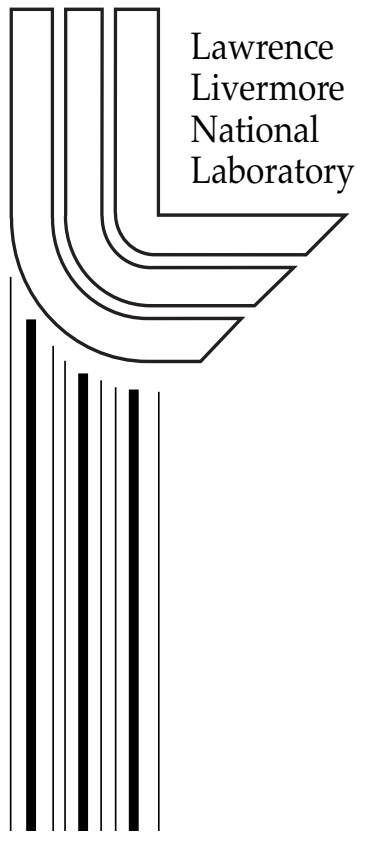




\section{DISCLAIMER}

This document was prepared as an account of work sponsored by an agency of the United States Government. Neither the United States Government nor the University of California nor any of their employees, makes any warranty, express or implied, or assumes any legal liability or responsibility for the accuracy, completeness, or usefulness of any information, apparatus, product, or process disclosed, or represents that its use would not infringe privately owned rights. Reference herein to any specific commercial product, process, or service by trade name, trademark, manufacturer, or otherwise, does not necessarily constitute or imply its endorsement, recommendation, or favoring by the United States Government or the University of California. The views and opinions of authors expressed herein do not necessarily state or reflect those of the United States Government or the University of California, and shall not be used for advertising or product endorsement purposes.

This work was performed under the auspices of the U. S. Department of Energy by the University of California, Lawrence Livermore National Laboratory under Contract No. W-7405-Eng-48.

This report has been reproduced directly from the best available copy.

Available electronically at http://www.doc.gov/bridge

Available for a processing fee to U.S. Department of Energy

And its contractors in paper from

U.S. Department of Energy

Office of Scientific and Technical Information

P.O. Box 62

Oak Ridge, TN 37831-0062

Telephone: (865) 576-8401

Facsimile: (865) 576-5728

E-mail: reports@adonis.osti.gov

Available for the sale to the public from

U.S. Department of Commerce

National Technical Information Service

5285 Port Royal Road

Springfield, VA 22161

Telephone: (800) 553-6847

Facsimile: (703) 605-6900

E-mail: orders@ntis.fedworld.gov

Online ordering: http://www.ntis.gov/ordering.htm

OR

Lawrence Livermore National Laboratory

Technical Information Department's Digital Library

http:/ / www.llnl.gov/tid/Library.html 


\title{
SIMULATING MARVEL WITH THE STUN CODE
}

\author{
L. A. Glenn ${ }^{\dagger}$
}

\begin{abstract}
MARVEL, a nuclear-driven shock-tube experiment, consisted of a 2.2 kiloton nuclear explosive detonated 176 meters underground at one end of a 122-meter long, 1-meter diameter horizontal tunnel. Vaporization of material in the immediate vicinity of the explosive provided the source of high-energy driver gas. The driven gas was the ambient atmospheric air in the tunnel. The event was staged as an experimental and calculational study of the time dependent flow of energy in the tunnel and surrounding alluvium. In this report we describe the derivation and implementation of a '1-3/4D' hydrocode to simulate the experiment. Calculations were performed to study the influence of energy transport to, and mass ablation from, the walls of the tunnel on the shock velocity.
\end{abstract}

\footnotetext{
${ }^{\dagger}$ Lawrence Livermore National Laboratory
} 


\section{INTRODUCTION}

The MARVEL event was a nuclear driven shock tube experiment. A 2.2 kt nuclear device was detonated at one end of a 1-meter diameter, 122-meter long horizontal underground tunnel that was excavated 176 meters below ground level in Yucca Flat at the Nevada Test Site. The purpose of the experiment, which took place on September 20, 1967, was to study the hydrodynamic flow of energy and debris down a tunnel from a nuclear explosion (Thomsen 1978). One of the prime measurements was the shock arrival time. Both piezoelectric crystal gages and slifers ${ }^{\dagger}$ were employed for this purpose and high quality data were obtained. Hydrodynamic simulations of the experiment were not in accord with the experimental results, the measured time-of-arrival (TOA) at the end of the tunnel being far less than predicted. Initial calculations showed that the discrepancy might be accounted for by taking into account wall ablation, but a detailed model for accomplishing this was beyond the state-of-the-art at the time.

Recent 2D calculations performed with the GEODYN (Lomov 1999) and RAPTOR (Howell and Greenough 1999) codes have confirmed the earlier simulations. Neither of these codes is capable of $a b$ initio description of the wall ablation process, which is driven mainly by radiation transport from the explosion products. The main problem derives from the fact that the mean free path for photon transport in the cold rock wall is estimated to be of the order of $10^{-7} \mathrm{~m}$. Accurate calculation of wall transport thus requires extremely high radial resolution. Constraints on the maximum zonal aspect ratio then translate into zone numbers that are impractically large, even for the massively parallel ASCI systems currently available. A sub-grid model of the ablation process has been devised and is in the process of being implemented and tested in the RAPTOR code. However, even with this simplification, the resulting calculations are expected to be quite time consuming. In what follows we describe the implementation of a still simpler model in the ' $1-3 / 4 D^{\prime} \quad S$ (phere and) TUN(nel) code. Much of the conceptual basis for the STUN code derives from earlier work by Glenn $(1982,1990)$.

$t_{\text {an }}$ electrical oscillator attached to a long piece of coaxial cable; the shock shorts out the cable, thereby changing the frequency of oscillation and giving a continuous record of shock position with time 


\section{MODEL DESCRIPTION}

A schematic of the MARVEL set-up is shown in figure 1.

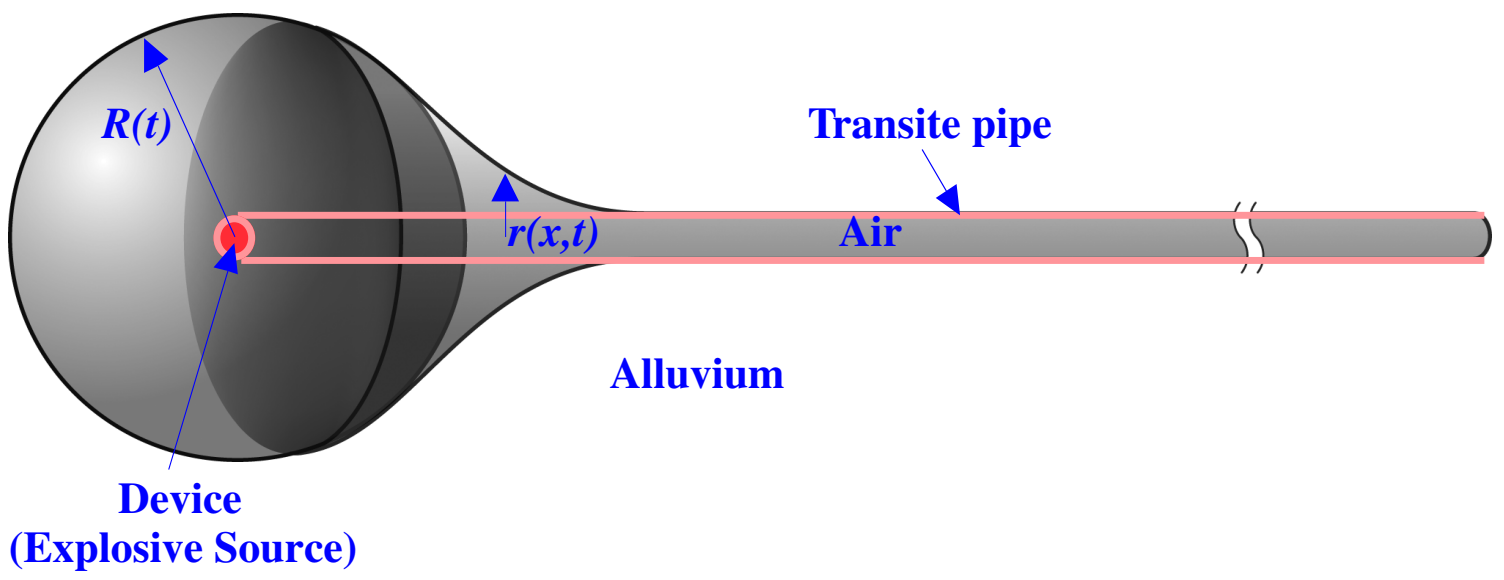

Figure 1. MARVEL set-up schematic. The initial configuration is defined in red. The shaded outline sketches the approximate geometry when the shock has just arrived at the end of the tunnel.

In the region immediately surrounding the explosive source the flow is essentially spherical, with explosion products (possibly surrounded and mixed with ambient air) forcing the surrounding rock into a cavity. The expanding cavity is connected to the tunnel, in which the main flow is essentially axial. To the extent that these simplifications prevail, the equations of motion describing this system can be written:

$$
\begin{gathered}
\frac{d(\rho A)}{d t}+\rho A \frac{\partial u}{\partial x}=\alpha S \phi / H_{v} \\
\frac{d u}{d t}+\frac{1}{\rho} \frac{\partial \sigma_{X}}{\partial x}+\frac{\left(\sigma_{X}-\sigma_{T}\right)}{\rho A} \frac{\partial A}{\partial x}+F u|u|=0 \\
\frac{d\left(e+q+\frac{1}{2} u^{2}\right)}{d t}+\frac{1}{\rho A} \frac{\partial\left(\sigma_{X} u A\right)}{\partial x}=0
\end{gathered}
$$

$\rho, u$, and $e$ are respectively the mass density, particle velocity and specific internal energy, and $\sigma_{X}$ and $\sigma_{T}$ are the axial and transverse components of stress. The operator

$$
\frac{d}{d t} \equiv \frac{\partial}{\partial t}+w \frac{\partial}{\partial x}
$$

and

$$
w=u-s
$$

$w$ is the particle velocity relative to a local coordinate frame moving with velocity $s(x, t)$, so that equations (1) - (3) define an Eulerian representation when $s=0$ and Lagrangian 
flow when $s=u$; in general $s$ is arbitrary and may be chosen to improve computational efficiency and accuracy. In particular, for the present application, material interfaces (source/air, air/rock, source/rock) are taken to be Lagrangian. An effective algorithm is to choose $s$ such that there is uniform resolution between Lagrangian faces by using accordion-like tesselation.

The flow cross section inside the rock walls is the key to the representation; specifically:

$$
A(x)= \begin{cases}4 \pi x^{2}, & \text { for } x \leq R(t) \\ \pi[r(x, t)]^{2}, & \text { for } x>R\end{cases}
$$

subject to the initial conditions: $r(x, 0)=R(0)=R_{0}$, where $R_{0}$ is the initial tunnel radius. The technique for estimating $R(t)$ and $r(x, t)$ is described later below.

$H_{v}$ in eq. (1) is the specific cohesive energy of the tunnel wall material, $S=2 \pi r$ is the wall surface per unit length, $\phi$ is the rate of energy delivered to the wall per unit area, and $\alpha$ is the fraction of the resultant ablated mass that is mixed with the flow.

The last term in equation (2) accounts approximately for wall drag in the tunnel, with the drag parameter defined as:

$$
F= \begin{cases}f / 4 r, & \text { for } x>R(t) \\ 0, & \text { otherwise }\end{cases}
$$

where the friction factor, $f$, is taken to be a function of the Reynolds number, based on the local diameter. For laminar flow $(R e=2 r u / v<2500 ; v$ is the kinematic viscosity), we use Stokes' law:

$$
f=64 / R e
$$

whereas when the flow is turbulent ( $R e \geq 2500$ ), which is almost always the case in the tunnel, the Prandtl-Karman relation is assumed to apply:

$$
f^{-1 / 2}=2 \log _{10}\left[R e f^{1 / 2}\right]-0.8
$$

Mach and Prandtl number effects on the wall drag are thought to be of lesser importance and were ignored.

The equation of state (EOS) has the general form:

$$
p=\left(\sigma_{X}+2 \sigma_{T}\right) / 3=p(\rho, e)
$$

The right-hand side of (9) may be analytic or tabular. In the calculations described below, LEOS tables were used throughout. The deviatoric stress components $s_{X}=\sigma_{X}-p$ and $s_{T}=\sigma_{T}-p$ are obtained from a constitutive model that accounts for pressure and strain hardening of the yield strength, porosity, melting, damage due to distortional deformation and many other effects (Rubin et al. 2000). 
The rate of energy per unit area delivered to the walls of the tunnel is simply:

$$
\phi= \begin{cases}\beta\left[h\left(T-T_{S}\right)+\frac{a c}{4} \epsilon\left(T^{4}-T_{S}^{4}\right)\right], & \text { for } x>R(t) \\ 0, & \text { otherwise. }\end{cases}
$$

$T_{S}$ is the surface temperature of the tunnel walls, $a$ is the radiation density constant, $c$ is the speed of light, and the emissivity coefficient, $\epsilon$, is approximated by:

$$
\epsilon=\left(\frac{3}{4} \rho K_{r} r\right)^{-1}
$$

where $K_{r}=K_{r}(\rho, T)$ is the Rosseland mean opacity. Equation (12) is appropriate in the diffusion approximation, i.e., where $\epsilon<<1$, but does not take into account strong crossflow (axial motion) near the wall. In this case, as will be seen below, it may only provide a lower bound for $\epsilon$, whose upper bound is the black body value of unity. The binary coefficient, $\beta$, takes the value 0 or 1 depending on whether or not energy transport to the walls is to be considered.

The convective heat transfer coefficient, $h$, consistent with eq. (9) is simply:

$$
h=0.0384 \rho c_{p} u\left(R e_{d}\right)^{-1 / 4}
$$

where $c_{p}$ is the specific heat. For $T>>T_{S}$, the convective term in eq. (11) is generally negligible, but it has been included for completeness.

With these definitions, the rate of energy transport to the walls per unit mass of flow in the tunnel is:

$$
\frac{d q}{d t}=\frac{2 \phi}{\rho r}
$$

To determine $R(t)$, we simply solve the equations of motion in spherical coordinates for the exploding source in a rock cavity, with $F=\phi=0$. The initial conditions for this problem are: $e=W / m_{S}, \rho=m_{S} /\left[(4 / 3) \pi r_{S}^{3}\right]$, where $W$ is the device yield (2.2 kt for MARVEL), $m_{S}$ is the mass of the device canister ( $\approx 1.6$ metric tons), and $r_{S} \leq R(0)$ is the source radius. For our purposes, the source is taken to be iron, which represents most of the canister/device mass. The initial pressure in the air and in the rock is ambient and the initial velocity is zero everywhere. To account for the presence of the tunnel, however, requires placing a mass sink at the expanding cavity radius, $\mathrm{R}(\mathrm{t})$, since source material and air escape the spherical system once the device explodes. The mass sink is provided by simultaneous solution of the ' $1-1 / 2 D^{\prime}$ variable area problem described by eq. 1-13 with the same initial conditions. The mass flow rate per unit area at $\mathrm{R}(\mathrm{t})$, $\rho(x=R, t) u(x=R, t)$, from this problem constitutes the mass sink for the spherical problem while the cavity boundary, $R(t)$, supplies the boundary condition for the axial flow problem. 
The model described thus far accounts for the motion of the cavity walls immediately surrounding the device, but not the walls of the cylindrical tunnel. The radial motion of the tunnel walls could be handled in a manner similar to that employed for the cavity (by simultaneously solving a set of 1D problems in cylindrical symmetry) but, as will be demonstrated below, this motion is less significant and a much simplified approach was used. In the strong-shock approximation, the amplitude of the velocity vector normal to the (tunnel) wall surface at time $t$ is:

$$
\dot{z}(x, t)=p(x, t) / \rho_{w} c_{s w} .
$$

Equation (15) will overpredict the wall motion at late times; to correct for this, the actual value of $\dot{z}$ employed was the minimum of the value derived from eq. (15) and that calculated from the momentum conserving expression:

$$
\int p(x, t) d t=\rho_{w} z \dot{z}
$$

where $\rho_{w}$ is the density of the rock and $c_{s w}$ is the shock velocity as determined from the Hugoniot. In the finite difference scheme, the implementation is via a split operator approach, similar to the manner in which eq. (11) above is employed to compute the wall ablation. For each wall node at time $t^{k}$ the wall velocity amplitude is evaluated via eqs. (15) and (16). Together with the stored wall coordinates, this allows the wall velocity vector to be calculated, the wall displacement components to be updated, and new zonal volumes to be computed. The interior fluid density is then recalculated and, finally, the $p d V$ work done by the fluid on the walls is incremented and stored and the specific internal energy of the fluid is decreased accordingly. It should be noted that a more complicated approach was adopted previously to analyze MARVEL (Crowley et al. 1970) in which a fully 2D axisymmetric code was employed to determine the tunnel boundary conditions, together with a quasi-1D solution of the axial flow in the tunnel. The present approach is much faster and produces comparable results.

\section{CALCULATIONS}

\section{Effect of Tunnel Wall Motion on Shock TOA}

Figure 2 shows the computed shock TOA with and without the implementation of equations (15) and (16), compared with the experimental results. In neither of these calculations was wall heating taken into account, so that $\beta$ was set to zero in eq. (11). It is clear that the motion of the tunnel wall downstream of the expanding cavity region has relatively little effect on the shock trajectory in the tunnel, thus justifying the approximate treatment of this motion. In both cases, the TOA at the end of the tunnel is far 
too short; with rigid tunnel walls, the shock arrives at the end of the tunnel in $0.83 \mathrm{~ms}$ as compared with $0.96 \mathrm{~ms}$ when the tunnel walls are allowed to respond to the internal pressure; this compares with $>4.0 \mathrm{~ms}$ in the MARVEL experiment (the last datum for MARVEL was $3.98 \mathrm{~ms}$ at $119.05 \mathrm{~m}$ ).

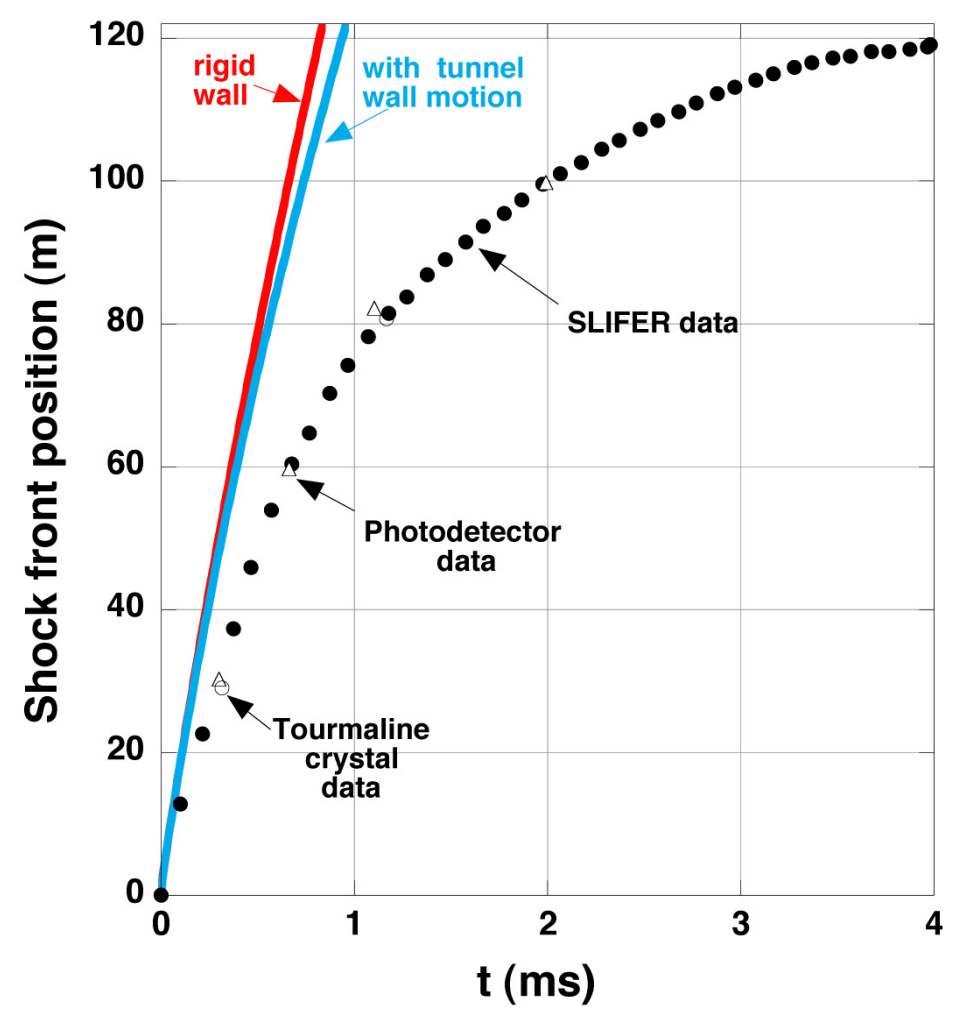

Figure 2. Computed shock position as a function of time compared with experimental data. Calculations are for the case where wall heating is ignored $(\beta=0)$.

\section{Effect of Energy Transport to the Tunnel Walls on Shock TOA}

Equations (1)-(15) describe an adiabatic system, wherein energy transported to the tunnel walls is returned via ablated wall mass. When $\alpha=0$, however, no ablation occurs; in this case the energy is lost from the tunnel system. Figure 3 compares the computed shock TOA, (for the cases when $\beta=1$ in eq. (11)), with $(\alpha=1)$ and without $(\alpha=0)$ ablation, to the calculation in Figure 2 , with $\beta=0$, and with the experimental results. In these calculations, and all that follow, tunnel wall motion is taken into account via equations (14) and (15).

Ignoring ablation for the moment, it is observed that the shock TOA at the end of the tunnel (EOT) increases from $0.96 \mathrm{~ms}$ to $1.02 \mathrm{~ms}$ when energy transport to the tunnel 
walls is allowed, i.e., there is virtually no change in the shock velocity, which is still far in excess of that experimentally observed.

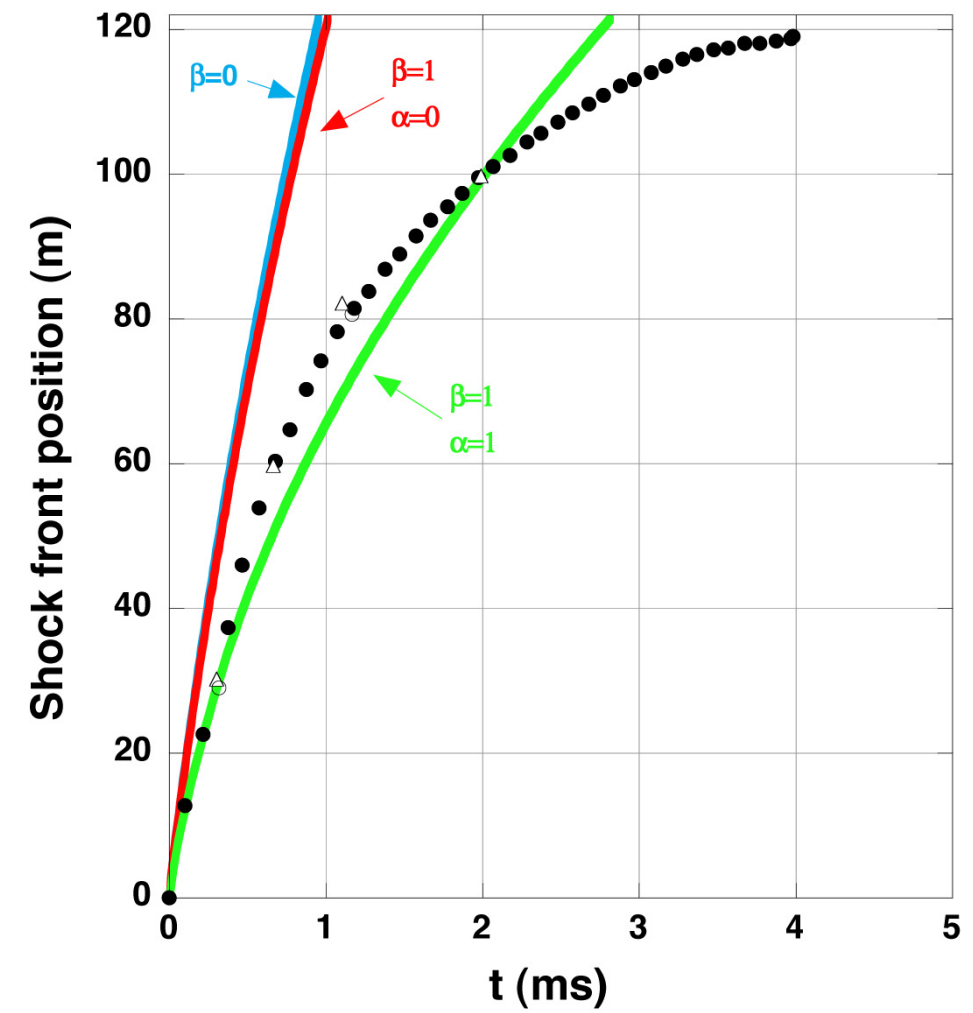

Figure 3. Computed shock position as a function of time with $(\beta=1)$ and without $(\beta=0)$ wall heating and with $(\alpha=1)$ and without $(\alpha=0)$ mass ablation. The symbols show the experimental data.

When the energy transport is fully converted to ablated wall mass and instantaneously mixed with the tunnel flow, the comparison with experiment is improved, with the TOA increasing to $2.81 \mathrm{~ms}$, but this still lags the data. One possible source of error is the opacity data, for which there is little experimental validation in the range of interest. Moreover, there are apparently several versions of LEOS opacity tables currently in use and a cursory check of the one we have been using for air, at the low end of this range $(\mathrm{T}=1.5 \mathrm{eV})$, showed that the Rosseland opacities were up to three orders of magnitude higher than those obtained from another extant version of LEOS.

\section{Effect of Opacity on Shock TOA}

Since there are very few experimental data and the theoretical models for opacity in the range of interest $\left(1<T<30 \mathrm{eV}, 10^{-3}<\rho<10^{-1} \mathrm{~g} / \mathrm{cm}^{3}\right)$ are not considered accurate, 
figure 4 shows the effect on the shock TOA of including an opacity multiplier, $\kappa$ in equation (12), defined by: $K_{r}=\kappa K_{r L E O S}$.

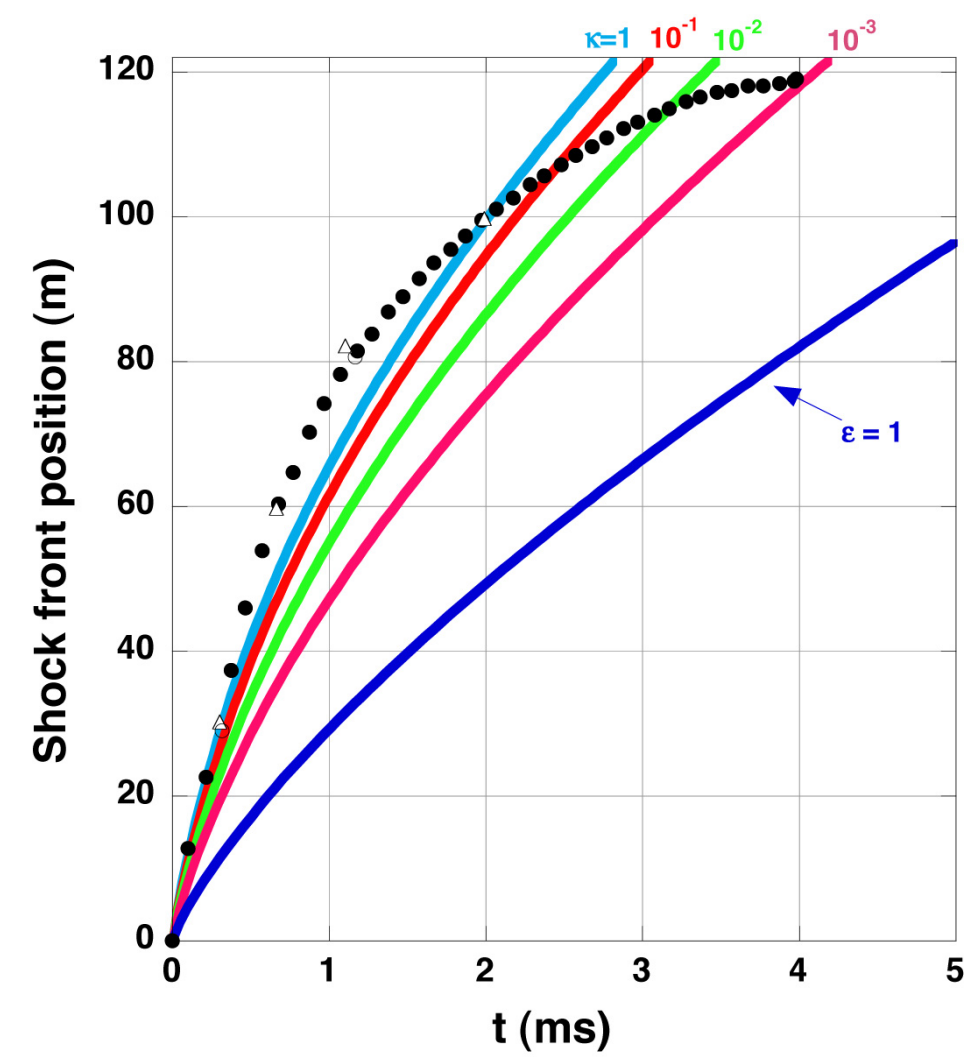

Figure 4. Computed shock position as a function of time for variable opacity multiplier, $\kappa$, and for emissivity, $\epsilon=1$. The symbols show the experimental data.

In this and subsequent figures, $\beta$ is taken to be unity. Although there is no reason to believe that a simple multiplier will account for any opacity errors, it is seen that as $\kappa$ decreases by decades from 1 to $10^{-3}$, the computed TOA at the EOT agrees much better with the experimentally observed value, although agreement with the shock velocity profile is still poor. Also shown on the figure is the solution obtained when the emissivity coefficient takes the limiting black body value of unity. This value might be approached if turbulent transport from the core flow to the wall were to diminish the radial temperature gradient established by the diffusion of radiant energy. In this case, the shock TOA is substantially retarded with respect to that of the data. As seen in the figure, the shock has moved only $96.4 \mathrm{~m}$ down the 122-m long tunnel by $5 \mathrm{~ms}$, at which point the calculation was terminated.

We emphasize that there are two uncertainties in the model, the value of the emissivity coefficient, $\epsilon$, and that of the mass fraction ablated, $\alpha$, that actually mixes with the core 
flow. Since heat transfer to the wall is dominated by radiation flow in equation (11), these two parameters actually appear as a product, $\xi=\alpha \epsilon$, in the equations of motion. In what follows we examine further the effect of varying this product on the shock TOA.

\section{Effect of Uniform Variation of the Parameter $\xi=\alpha \epsilon$ on Shock TOA}

Figure 5 shows the shock position as a function of time when $\xi$ is varied over the full range from 0 to 1 . It is observed that as $\xi$ is decreased from 1 to 0.5 the shock TOA at the end of the tunnel increases slightly and then, as $\xi$ is decreased further to 0.1 and then 0.01 , the tunnel-end shock TOA sharply decreases. The reason for the initial increase in the range $0.5<\xi \leq 1$ is the greater tunnel surface involved in the ablation process in this case. When $\xi=1$, the extremely high temperatures at the entrance of the tunnel induce very rapid ablation in this region which, in turn, cools the plasma and inhibits further ablation downstream. When $\xi$ is decreased to 0.5 , the initial ablation is less rapid, allowing more material from the wall to interact with the plasma at downstream locations. This effect is eventually offset when $\xi$ is reduced further.

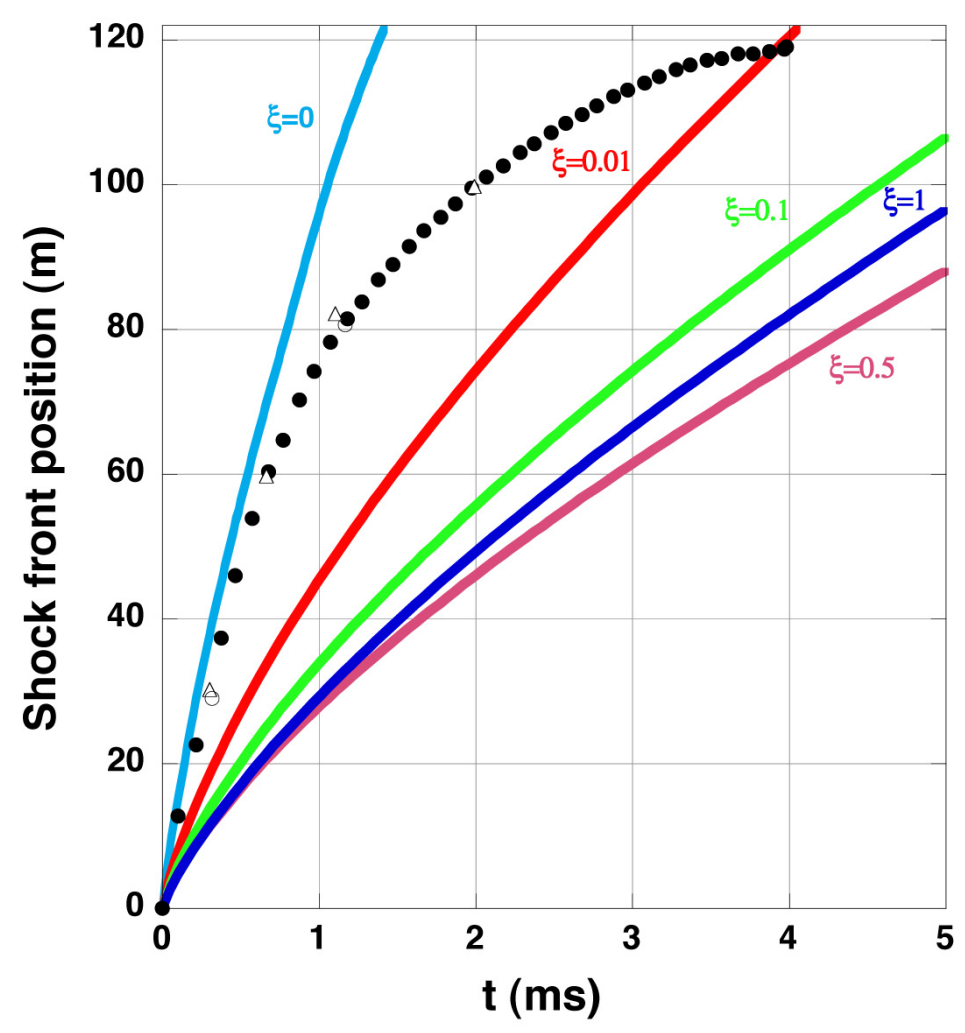

Figure 5. Computed shock position as a function of time with wall heating $(\beta=1)$ and ablation, for fixed values of the parameter $\xi=\alpha \epsilon$. The symbols show the experimental data. 
The best agreement with the experimentally observed shock arrival time at the end of the tunnel is seen to be for $\xi=0.01$. However, as in the previous figure, agreement with the shock velocity profile is poor. The data show the shock velocity to initially increase much more rapidly (in the first $60 \mathrm{~m}$ of tunnel) than exhibited in the calculations (except for $\xi=0$ ), and then decrease much more rapidly (in the remainder of the tunnel). In the last $10 \mathrm{~m}$, for example, the calculated average shock velocity with $\xi=0.01$ was $13.4 \mathrm{~km} / \mathrm{s}$, whereas the data indicate the actual value was $7.18 \mathrm{~km} / \mathrm{s}$. Moreover, the rate of deceleration exhibited in the experimental data near the tunnel-end is much greater.The "instantaneous" end-of-tunnel shock velocity was $2.02 \mathrm{~km} / \mathrm{s}$ as determined in the experiment, as compared with $10.8 \mathrm{~km} / \mathrm{s}$ in the calculation with $\xi=0.01$.

\section{Effect of Non-Uniform Variation of the Parameter $\xi=\alpha \epsilon$ on Shock TOA}

The data suggest that material ablated from the walls does not mix instantaneously with the plasma in the tunnel. Indeed, there is little reason to expect such behavior. 1D radiation-coupled hydrodynamic calculations of the region near the wall (radial flow only) show the time for the ablation products to fill the tunnel, based on the ablation velocity at the wall, to be small compared with the axial transit time of the shock. Therefore, free-stream turbulent transport must be the main mechanism for moving the ablation products into the flow. Although we are unable to model this process with the

STUN code (since one of the basic assumptions in our simplified approach was that the flow in the tunnel is strictly axial), we can easily implement a non-uniform mixing algorithm. The results displayed in figure 5 suggest a pressure dependence for the mixing rate that would require longer times for mixing when the pressure in the tunnel is high than when the pressure is low. After some trial and error, we found that the function:

$$
\xi=\min \left(\frac{c_{1}+c_{2} / p^{n}}{p}, 1\right)
$$

where $c_{1}=2.3 \times 10^{-3} \mathrm{GPa}, c_{2}=1 ., n=6$, and $p$ is in GPa, gives a reasonably good representation of the experimental data. Figure 6 plots the calculated shock position as a function of time when eq. (17) is used. It is observed that, although the calculation generally agrees with the experimental results, the calculated final shock velocity is still too high. Nevertheless, the non-uniform mixing simulation closely approximates the data over most of the tunnel length.

Figure 7 compares the pressure, density, temperature, and particle velocity profiles in the tunnel at the time when the shock just arrives at the closed end for the two cases: $\beta=0$ (no energy transport to the walls) and $\beta=1$ with the parameter, $\xi$, defined by equation (17), as represented in figure 6 . In the former case, as noted in figure 3 , shock 
arrival at the EOT occurs at a time of $0.96 \mathrm{~ms}$, compared with $3.6 \mathrm{~ms}$ in the latter case.

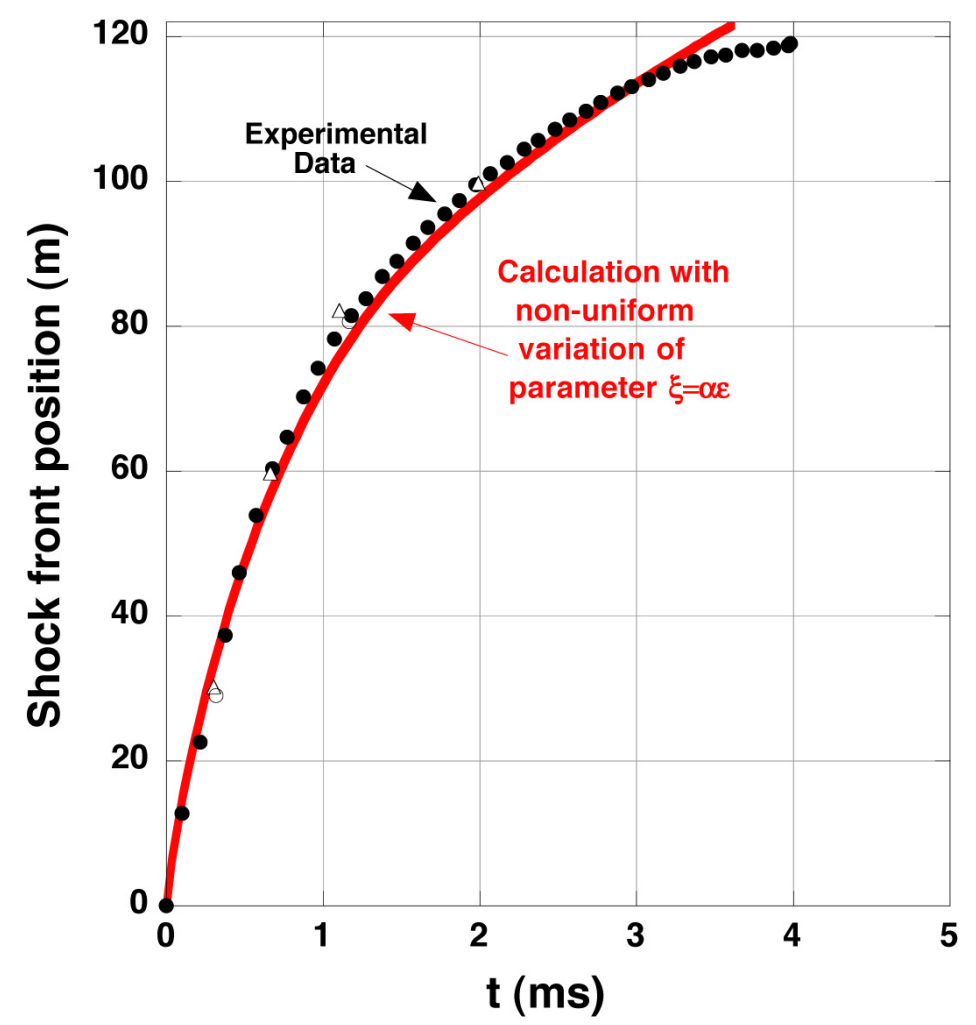

Figure 6. Computed shock position as a function of time with wall heating and ablation and the parameter, $\xi$, defined by equation (17).

The addition of the ablated mass causes the temperature at the end of the tunnel to drop from 36 to $2.2 \mathrm{eV}$ and the particle velocity from 83 to $11.5 \mathrm{~km} / \mathrm{s}$. At $3.6 \mathrm{~ms}$, the overall ablated mass in the non-uniform mixing case was 30\% in excess of the initial source mass (device plus canister) and more than 20 times the initial mass of air in the tunnel. At this same time, the cavity radius, $R$, had grown to $4.97 \mathrm{~m}$, almost 10 times the initial tunnel radius, and was still expanding at a rate of $700 \mathrm{~m} / \mathrm{s}$.

Figure 8 depicts the pressure and impulse per unit area experienced by the closed endof-tunnel (EOT) for the 2 cases discussed above in figures 6 and 7 . The peak pressure of 34.1 GPa for $\beta=0$ compares with the value of $1.62 \mathrm{GPa}$ for $\beta=1$, non-uniform mixing and parameterized emissivity, and the impulse applied to the EOT wall is similarly greater. Clearly, in an actual tunnel complex, mass ablation from the walls will have a 
major impact on the response and resistance of any blast doors that are employed.
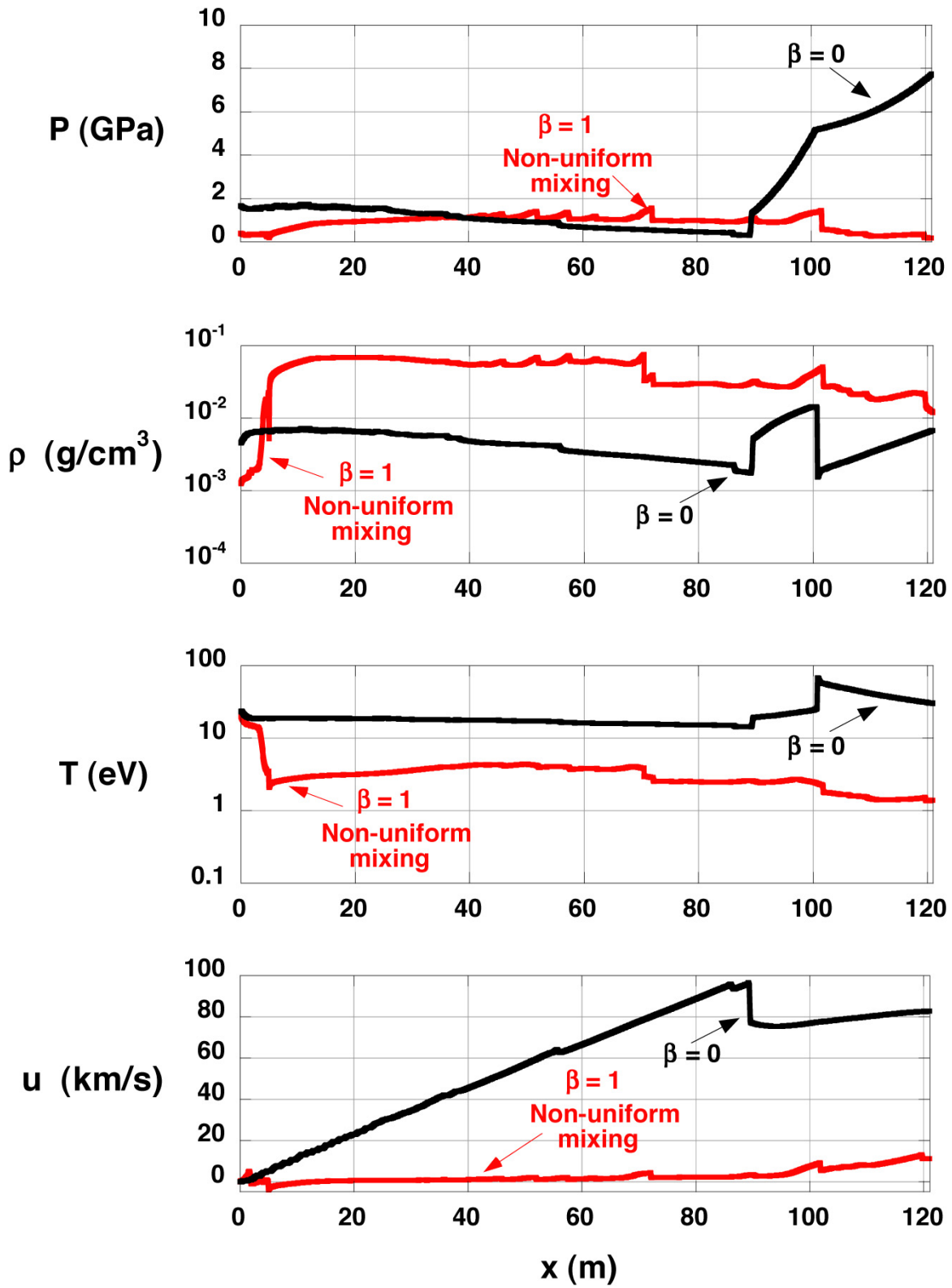

Figure 7. Computed pressure, density, temperature and particle velocity profiles when the shock has arrived at the end of the tunnel for the cases $\beta=0$ and $\beta=1$ and the parameter, $\xi$, defined by equation (17). 

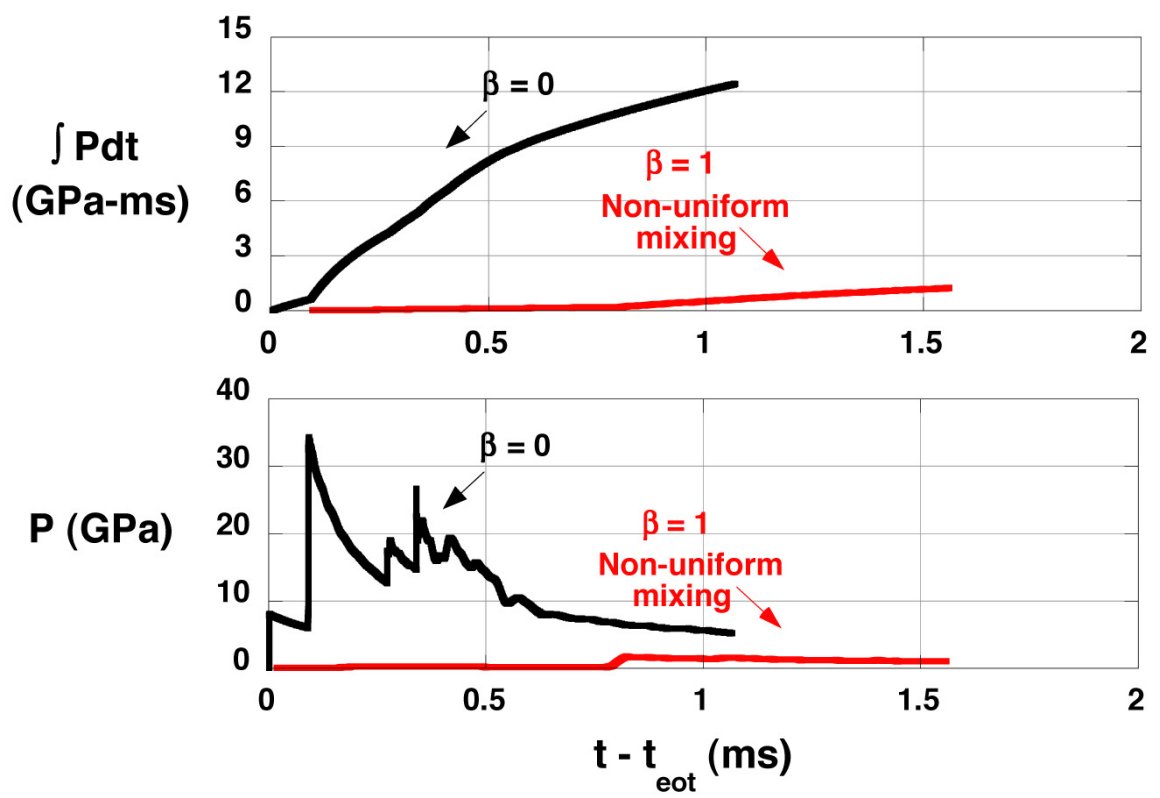

Figure 8. Computed pressure and impulse per unit area on the closed end-of-tunnel as a function of time from shock arrival for the cases $\beta=0$ and $\beta=1$ and the parameter, $\xi$, defined by equation (17).

\section{CONCLUDING REMARKS}

We have shown that there are two significant areas of uncertainty that need clarification before accurate $a b$ initio modeling of bomb-in-tunnel scenarios will be possible. First, experiments and/or improvements in theoretical models are needed to provide accurate opacities for air and other relevant source region material in the so-called 'low-temperature' regime, i.e., $1<T<30 \mathrm{eV}$ and $10^{-4}<\rho<1 \mathrm{~g} / \mathrm{cm}^{3}$. Second, a better understanding of the mechanism of turbulent transport of ablated material from the tunnel wall boundary layer is required.

Despite these limitations, we have demonstrated that a relatively simple model can account for the shock velocity measurements that were obtained in the MARVEL event. This model has been incorporated into a '1-3/4D' hydrocode called S(phere and) TUN(nnel) that permits detailed evaluation of the environment in the tunnel as a function of time. Moreover, the STUN code has provided insight into the modeling requirements for more accurate (2D/3D) representation of the ablation and turbulent mixing processes that must have occurred in the event.

There are two improvements that should be made to increase the usefulness of the code for scaling and effects studies. First, provision should be made to account for 
multiple blast doors that might be placed in an actual tunnel complex. (In the STUN context, a blast door would simply be a Lagrangian mass point that functions like a burst diaphragm, i.e., a rigid wall of the appropriate areal density that releases once a threshold pressure has built up on the upstream face). Next, account should be taken of nonlinear tunnels, i.e., tunnels with sharp bends, since these are likely to be employed as momentum traps to minimize damage in case of attack. Such bends are easily handled in the STUN context by employing a quasi-rigid wall reservoir zone at the bend location, which aligns the velocity vector of the tunnel flow with the wall vector and provides a pressure accumulator to account for the concomitant momentum loss. Both of these modifications will be implemented in the near future.

\section{REFERENCES}

Crowley, B. K., Glenn, H. D., and R. E. Marks, An Analysis of MARVEL - A Nuclear ShockTube Experiment, Lawrence Livermore National Laboratory Report UCRL-72489, Rev. 1, January 14, 1970.

Glenn, L. A., Blast Attenuation in a Shot Tunnel using Water Jets, Lawrence Livermore National Laboratory Report UCRL-19419, July 2, 1982.

Glenn, L. A., Design Limitations on Ultra-Hight Velocity Projectile Launchers, Int. J. Impact Engrg, 10, 185-196, 1990.

Howell, L. H. and J. A. Greenough, A Block Structured Adaptive Mesh Refinement Algorithm for Diffusion Radiation, Lawrence Livermore National Laboratory Report UCRL133094, 1999.

Lomov, I.N., Theory and Implementation of the GEODYNE Code, Lawrence Livermore National Laboratory Report, in preparation.

Rubin, M. B., Lomov, I. N., Grieger, J., Glenn, L. A., Antoun, T. H., and J. P. Morris, Material Model in the GEODYNE Computer Code, Lawrence Livermore National Laboratory Internal Report, September 1, 2000.

Thomsen, J. M., MARVEL Revisited: Experiment Design and Data Analysis, Lawrence Livermore National Laboratory Report UCRL-52445, February 28, 1978. 